\title{
Schimmelpilzinfektion der Lunge
}

Eine Forschungsgruppe der Medizinischen Universität Graz stellte sich die Frage, wie man der mit COVID-19 assoziierten pulmonalen Aspergillose begegnen kann.

Schimmelpilzinfektionen stellen laut Intensivmedizinern ein zunehmendes Problem bei COVID-19 Patienten dar. „Aktuell sorgt die sogenannte "Black Fungus Pandemie" bei indischen COVID-19 Patienten für zunehmende Sorge bei globalen Gesundheitsbehörden", so Prof. Dr. Robert Krause, supplierender Leiter der Klinischen Abteilung für Infektiologie an der Med Uni Graz. "Black Fungus" steht für eine fungale Superinfektion mit Schimmelpilzen aus dem Genus der Mucorales spp.

Zudem ist bekannt, dass COVID-19-Patienten, welche diese Superinfektion entwickeln, besonders schlechte Überlebenschancen zeigen. Warum es gerade bei Bewohnern des indischen Subkontinents zu diesen besorgniserregenden Entwicklungen kommt ist nicht restlos geklärt. Krause: „In der Debatte stehen vor allem das feuchtwarme Klima der Tropen, die schlechte Gesundheitsversorgung der Bevölkerung speziell in Bezug auf chronische Erkrankungen wie Diabetes, sowie auch der immer breitere Einsatz von notwendigen Korticosteroiden in der Behandlung von COVID-19."

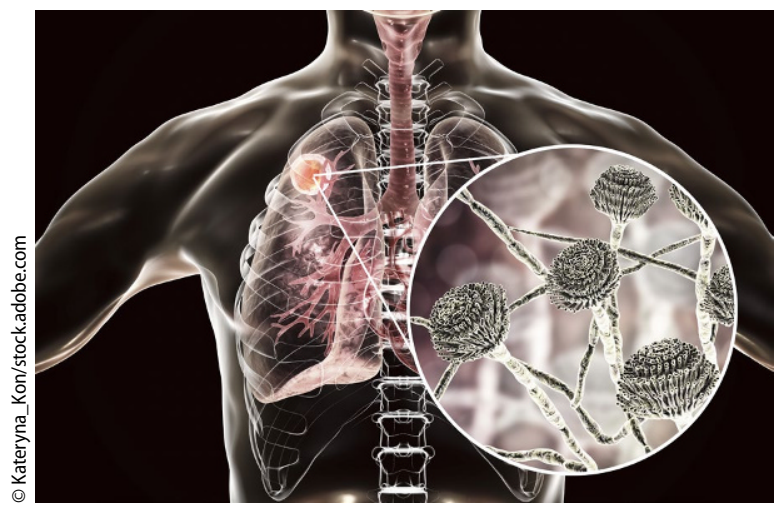

Abb. 1 A Infektionen mit Aspergillus sind vor allem bei intensivpflichtigen COVID-19 Patienten ein zunehmendes Problem
Auch in Europa stellen Schimmelpilzinfektionen, vor allem bei intensivpflichtigen COVID-19 Patienten, ein zunehmendes Problem dar (• Abb. 1). In der Gruppe der Patienten der westlichen Welt sind jedoch in den seltensten Fällen Mucorales-Pilze das Problem, sondern Aspergillus. Aufgrund dieser Problematik hat sich bereits ein eigenes Krankheitsbild entwickelt - die COVID-19 assoziierte Pulmonale Aspergillose (CAPA).

\section{Erkrankung mit lebens- \\ bedrohlichen Folgen}

„Im Rahmen unserer Forschungsarbeit haben wir alle intensivpflichtigen COVID-19-Fälle, welche an der Med Uni Graz bzw. dem LKH-Universitätsklinikum Graz behandelt wurden, untersucht. Wir konnten erstmals in Österreich eine konkrete Inzidenzzahl für die CAPA im Intensivbereich definieren", fasst Doz. Dr. Stefan Hatzl von der klinischen Abteilung für Hämatologie zusammen. Auf den Intensivstationen am Universitätsklinikum Graz haben $17 \%$ der Patienten zumindest ein diagnostisches Kriterium hinsichtlich einer CAPA entwickelt. Die Diagnose der CAPA wurde im Durchschnitt 6 Tage nach der Intensivstationsaufnahme gestellt.

COVID-19-Patienten verstorben, zusätzlich konnten wir auch zeigen, dass CAPA ein unabhängiger prognostischer Parameter für den Tod der COVID-19 Patienten war", fasst Prof. Dr. Gernot Schilcher (Universitätsklinik für Innere Medizin, Graz) zusammen.

\section{Pilzprophylaxe}

Das Hauptaugenmerk der vorliegenden Arbeit lag auf dem Verhindern dieser prognoselimitierenden Erkrankung im Sinne der Prophylaxe einer CAPA. „Wir konnten in unserer Studie zeigen, dass die prophylaktische Verabreichung eines Pilzmedikaments mit definierter Wirkung gegen Schimmelpilze CAPA-Fälle verhindern konnte. In der Gruppe von Patienten mit Pilz-Prophylaxe entwickelten nur $2 \%$ der Patienten eine CAPA verglichen mit $17 \%$ in der Gruppe ohne Pilz-Prophylaxe", so Krause.

\section{Literatur}

1. Hatzl S et al (2021) Antifungal prophylaxis for prevention of COVID-19-associated pulmonary aspergillosis in critically ill patients: an observational study. Crit Care 25(1):335. https://doi.org/10.1186/s13054021-03753-9.

》) Nach der Diagnose einer Schimmelpilzinfektion sind $87 \%$ der COVID-19-Patienten verstorben

„In einem nächsten Schritt untersuchten wir die Auswirkungen einer CAPA-Diagnose auf die Prognose der Patienten. Wir konnten zeigen, dass nahezu alle Patienten, die eine CAPA entwickelt haben, verstorben sind: Nach der Diagnose einer Schimmelpilzinfektion sind $87 \%$ der
Hinweis des Verlags. Der Verlag bleibt in Hinblick auf geografische Zuordnungen und Gebietsbezeichnungen in veröffentlichten Karten und Institutsadressen neutral.

rheuma plus $2021 \cdot 20: 260$ https://doi.org/10.1007/s12688-02100475-2

(c) Springer-Verlag GmbH Austria, ein Teil von Springer Nature 2021 